\title{
EXEHDA-RR: Uma proposta para tratar incertezas e otimizar o processo de classificação de recursos na IoT
}

\author{
Renato Dilli, Amanda Argou, Ana Pernas, Renata Reiser, Adenauer Yamin \\ ${ }^{1}$ Universidade Federal de Pelotas (UFPel) \\ Rua Gomes Carneiro, 1 - Centro - CEP 96010-610 - Pelotas - RS - Brasil \\ \{renato.dilli, aacardozo, marilza, reiser, adenauer\}@inf.ufpel.edu.br
}

\begin{abstract}
The Internet of Things (IoT) is characterized by a broad range of resources connected to the Internet, requesting and providing services simultaneously. Given this scenario, suitably selecting the resources that best meet users' demands has been a relevant and current research challenge. This paper presents the EXEHDA-RR, a proposal to classify and select the most appropriate resource, applying fuzzy logic to solve uncertainties in the definition of ideal weights for QoS attributes, and adding machine learning to the preclassification of resources in order to reduce the computational cost generated by the MCDA algorithms. The experimental results of the pre-classification show the efficiency of the proposed model.
\end{abstract}

Resumo. A Internet das Coisas (IoT) é caracterizada por uma ampla gama de recursos conectados à Internet, solicitando e fornecendo serviços simultaneamente. Diante desse cenário, selecionar adequadamente os recursos que melhor atendam às demandas dos usuários tem sido um desafio de pesquisa relevante e atual. Este artigo apresenta o EXEHDA-RR, uma proposta para classificar e selecionar o recurso mais apropriado, aplicando lógica fuzzy para resolver incertezas na definição de pesos ideais para os atributos de QoS, e agregando aprendizado de máquina à pré-classificação dos recursos visando reduzir o custo computacional gerado pelos algoritmos MCDA. Os resultados experimentais da pré-classificação mostram a eficiência do modelo proposto.

\section{Introdução}

O cenário atual contabiliza aproximadamente 27 bilhões de coisas conectadas à Internet disponibilizando serviços aos clientes e previsão de mais de 125 bilhões até 2030 [IHS Markit 2017]. O número de serviços será maior à medida que as coisas físicas poderão fornecer uma ou mais funcionalidades.

Um desafio a ser vencido após a descoberta dos recursos é classificar os serviços para selecionar o que melhor atende a solicitação do usuário [García 2012], sendo esta uma tarefa difícil e demorada. Os processos de classificação vem se concentrando nas preferências do usuário, que frequentemente estabelecem uma ordem baseada na Qualidade de Serviço (QoS) ou propriedades não-funcionais (NFPs - Non-Functional Properties) [Schröpfer et al. 2006].

\footnotetext{
${ }^{*}$ O presente trabalho foi realizado com apoio da CAPES (Programa Nacional de Cooperação Acadêmica - Procad) e da FAPERGS (Programa Pesquisador Gaúcho - PqG).
} 
Os recursos, geralmente disponibilizados por meio de serviços, possuem atributos de QoS com valores apresentando unidades de medidas heterogêneas e faixas amplas nos valores mínimos e máximos. Para selecionar o recurso mais oportuno para a requisição do cliente, é essencial a correta especificação destes valores.

Os responsáveis por definir estes valores devem ter conhecimento de todas as características dos atributos de qualidade, e desta forma avaliar os valores para qualificar os recursos. Considerando que em cada domínio a definição destes valores pode ser feita por especialistas com percepções diferentes, surge a incerteza quando da definição dos valores a serem adotados como atributos de QoS.

Com a premissa de atender estes desafios, o objetivo geral deste trabalho é a concepção de um modelo para a classificação de recursos, denominado EXEHDA-RR (Resource Ranking). O trabalho tem como premissa contribuir com a arquitetura de software existente no serviço de descoberta do middleware EXEHDA.

O diferencial dessa proposta está no uso combinado de lógica fuzzy e aprendizado de máquina no reconhecimento de padrões para classificação de recursos. Os recursos são inicialmente classificados por algoritmo de Análise de Decisão Multicritério (MCDA). Em cada classificação, um novo treinamento do algoritmo de aprendizado de máquina é realizado para pré-classificar novos recursos à medida que eles entram na infraestrutura computacional. Esse processo reduz a necessidade de processar todos os cálculos do algoritmo MCDA em cada solicitação do cliente. O cenário de avaliação do EXEHDARR mostrou resultados satisfatórios pela combinação da lógica fuzzy com o aprendizado de máquina. Considerando a revisão de literatura e as demandas do grupo de pesquisa, os resultados alcançados são oportunos para uso no middleware EXEHDA.

Este artigo está organizado da seguinte forma: na Seção 2 é apresentada a arquitetura e as funcionalidades do serviço de descoberta e ranqueamento de recursos EXEHDARR, na Seção 3 o tratamento da incerteza na definição dos pesos de QoS é apresentado, na Seção 4 é apresentada a avaliação da acurácia da pré-classificação de recursos do EXEHDA-RR, na Seção 5 são discutidos os Trabalhos Relacionados e na Seção 6 estão as Considerações Finais.

\section{EXEHDA-RR: Arquitetura e Funcionalidades}

O EXEHDA [Davet 2015] é o middleware, foco deste trabalho, responsável por prover a infraestrutura computacional básica para a IoT. Este ambiente computacional é constituído por células de execução, nas quais os dispositivos computacionais são distribuídos. Cada célula é constituída dos seguintes componentes: (i) EXEHDABase, o elemento central da célula, sendo responsável por todos serviços básicos e constituindo referência para os demais elementos; (ii) o EXEHDANodo que corresponde aos dispositivos computacionais responsáveis pela execução das aplicações; (iii) o EXEHDANodo móvel, um subcaso do anterior, que corresponde aos dispositivos tipicamente móveis que podem se deslocar entre as células do ambiente ubíquo; (iv) o EXEHDABorda, responsável por fazer a interoperação entre os serviços do middleware e os diversos tipos de gateways; e (v) o EXEHDAGateway, que consiste no elemento responsável por setorizar pontos de coleta e/ou atuação distribuídos, disponíveis no meio físico, realizando a interação destes com os outros componentes do middleware.

O EXEHDA-RR (Resource Ranking) é um serviço para classificação de recursos 
proposto para o middleware EXEHDA. Sua arquitetura de software foi modelada considerando a dinamicidade em que os recursos entram e saem do ambiente, portanto, controla a presença de dispositivos, inclusive com baixo poder computacional, conectados à gateways. O aumento da expressividade na descrição e consulta por recursos foi provida através de tecnologias de Web Semântica com intuito de potencializar a descoberta de recursos no ambiente. A arquitetura de software do EXEHDA-RR (Figura 1) possui três componentes distintos: (CD) Componente Diretório; (CR) Componente Recurso; e (CC) Componente Cliente. O CD, localizado no EXHDABase, contém os módulos responsáveis pela descoberta e ranqueamento de recursos.

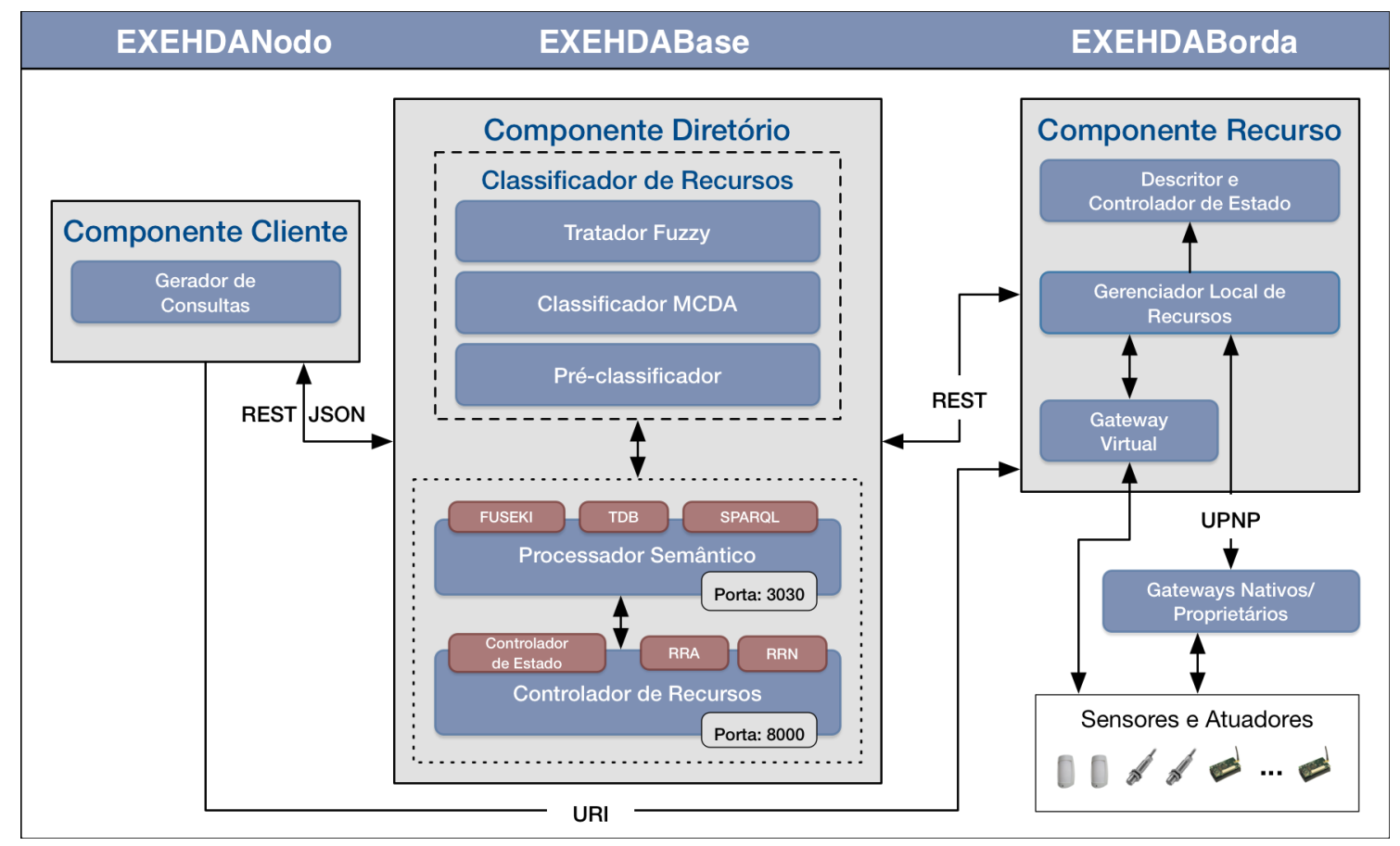

Figura 1. Sub-módulos do Classificador de Recursos

O processo de classificação dos recursos já descobertos é promovido pelo módulo "Classificador de Recursos". Este módulo contém os seguintes sub-módulos:

- Tratador Fuzzy. Tem por objetivo resolver as divergências entre vários especialistas na atribuição dos pesos de importância de cada atributo de QoS. As funções de pertinência empregadas e o método de defuzzificação estão detalhados em [Argou et al. 2017].

- Classificador MCDA. Responsável por classificar todos os recursos descobertos através do algoritmo MCDA proposto, tendo como entrada os pesos definidos pelo Tratador Fuzzy. O algoritmo MCDA proposto para classificação dos recursos é detalhado em [Dilli et al. 2017].

- Pré-classificador. Os novos recursos recém descobertos são pré-classificados através de algoritmo de aprendizagem de máquina, visando reduzir o custo computacional gerado pelo algoritmo MCDA.

\section{Tratamento da incerteza na definição dos pesos de QoS}

O EXEHDA-RR utiliza ontologias para descrição dos recursos e seus atributos de qualidade (QoS), avalia os requisitos não-funcionais dos recursos por meio de algoritmo 
MCDA, considera as preferências do cliente, trata a incerteza na definição dos pesos dos atributos através de lógica fuzzy e realiza a pré-classificação de recursos através do algoritmo de aprendizagem de máquina LMT.

No processo de classificação de recursos, que pode satisfazer a solicitação do usuário, a avaliação dos atributos de QoS é uma etapa desafiadora. A definição do grau de importância dos atributos de QoS pelo usuário e administradores da infraestrutura computacional é uma atividade que depende da experiência e do conhecimento de cada um. $\mathrm{O}$ tratamento da incerteza introduzida por essas divergências é uma das contribuições deste trabalho.

Para avaliar o emprego da lógica fuzzy na especificação dos atributos de QoS ideais, definidos pelos especialistas do ambiente computacional, foi utilizado o dataset QWS versão 2.0 disponibilizado por [Al-Masri and Mahmoud 2007], com 2.505 recursos e 9 atributos de qualidade. A Tabela 1 descreve os 5 atributos utilizados.

Tabela 1. Atributos do dataset QWS

\begin{tabular}{lll}
\hline Atributo & Descrição & Unid \\
\hline Response Time & Tempo do envio de uma requisição e receber sua resposta & $\mathrm{ms}$ \\
Availability & Número de invocações corretas/total invocações & $\%$ \\
Throughput & Total de invocações por um determinada período & $\%$ \\
Reliability & Taxa de erro no total de mensagens & $\%$ \\
Latency & Tempo que o servidor leva para processar uma requisição & $\mathrm{ms}$ \\
\hline
\end{tabular}

A Figura 2 e a Tabela 2 exibem os Termos de Linguísticos e os pesos atribuídos.

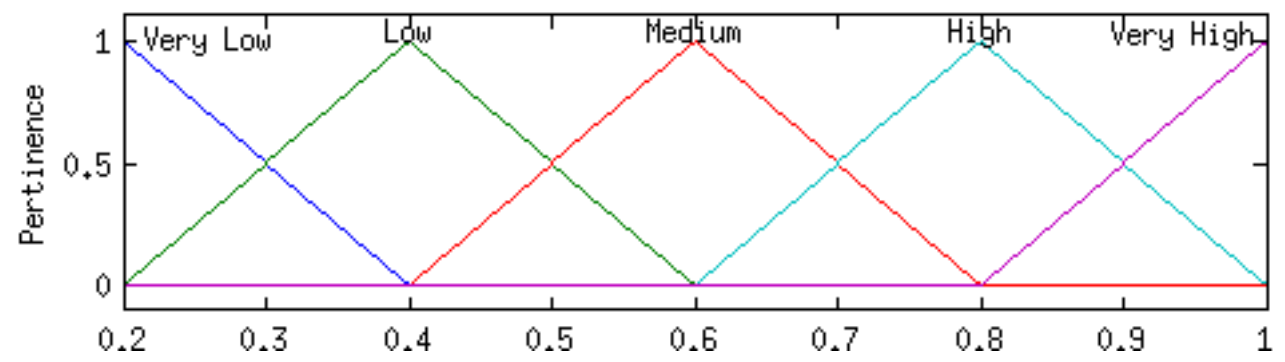

Figura 2. Representação dos conjuntos Fuzzy

Tabela 2. Grau de Importância

\begin{tabular}{ll}
\hline Termo Linguístico & Valor \\
\hline VH - Very High & $(0.8,1.0,1.0)$ \\
H - High & $(0.6,0.8,1.0)$ \\
M - Medium & $(0.4,0.6,0.8)$ \\
L - Low & $(0.2,0.4,0.6)$ \\
VL - Very Low & $(0.0,0.2,0.4)$ \\
\hline
\end{tabular}

\begin{tabular}{ccccc}
\multicolumn{5}{c}{ Tabela 3. Avaliação dos especialistas } \\
\hline Atrib & Esp1 & Esp2 & Esp3 & Esp4 \\
\hline RT & M & H & L & H \\
AV & VH & VH & H & VH \\
TH & M & M & H & H \\
RE & H & H & VH & H \\
LA & VH & H & VL & H \\
\hline
\end{tabular}

A Tabela 3 simula a definição do grau de importância de cada atributo de qualidade usando os Termos Linguísticos definidos por cinco especialistas. Esta avaliação visa 
definir os pesos a serem atribuídos a cada atributo, fornecendo os recursos mais adequados à solicitação do usuário.

Na Tabela 4 é apresentada a conversão dos Termos Linguísticos, atribuídos pelos especialistas, em números fuzzy triangulares.

A média difusa entre os especialistas e a defuzzificação usando o método da média ponderada também é apresentada. No final, os valores dos atributos são normalizados.

Tabela 4. Cálculo dos pesos de QoS

\begin{tabular}{llllllll}
\hline Atrib Esp1 & Esp2 & Esp3 & Esp4 & Méd.Fuzzy & Def & Norm \\
\hline RT & $(0.4,0.6,0.8)$ & $(0.6,0.8,1.0)$ & $(0.2,0.4,0.6)$ & $(0.6,0.8,1.0)$ & $(0.45,0.65,0.85)$ & 0.65 & 0.70 \\
AV & $(0.9,1.0,1.0)$ & $(0.9,1.0,1.0)$ & $(0.6,0.8,1.0)$ & $(0.9,1.0,1.0)$ & $(0.83,0.95,1.00)$ & 0.93 & 1.00 \\
TH & $(0.4,0.6,0.8)$ & $(0.4,0.6,0.8)$ & $(0.6,0.8,1.0)$ & $(0.6,0.8,1.0)$ & $(0.50,0.70,0.90)$ & 0.70 & 0.75 \\
RE & $(0.6,0.8,1.0)$ & $(0.6,0.8,1.0)$ & $(0.9,1.0,1.0)$ & $(0.6,0.8,1.0)$ & $(0,68,0.85,1.00)$ & 0.87 & 0.94 \\
LA & $(0.9,1.0,1.0)$ & $(0.6,0.8,1.0)$ & $(0.0,0.2,0.4)$ & $(0.6,0.8,1.0)$ & $(0,53,0.70,0.85)$ & 0.70 & 0.75 \\
\hline
\end{tabular}

Os valores normalizados resultantes serão aplicados no processo de classificação e seleção de recursos do algoritmo MCDA.

\section{Avaliação da acurácia na pré-classificação}

Nesta seção é avaliada a acurácia na classificação realizada pelo algoritmo de aprendizagem de máquina no processo de pré-classificação de novos recursos.

Para a realização dos testes foi utilizado o dataset QWS [Al-Masri and Mahmoud 2007], com os cinco atributos já apresentados na Tabela 1.

Para prototipação dos algoritmos de árvores de decisão, foi utilizado o MOA Massive Online Analysis. O MOA é um framework para mineração de dados. As bibliotecas do MOA e do WEKA foram desenvolvidas em java, portanto foi utilizado o Jpype para que o Python possa manipular as bibliotecas em Java.

\subsection{Explorando MCDA na construção do ranking de referência}

A aplicação do algoritmo MCDA é demonstrada na classificação de um dataset contendo 5 atributos de qualidade para 10 recursos. Cada linha representa um recurso e cada coluna um atributo de qualidade (Tabela 5). Os valores dos atributos Resp.Time e Latency são melhores se forem baixos e os valores dos atributos Availability, Throughput e Reliability são melhores se forem altos.

A primeira etapa da classificação é a normalização dos dados. Para tanto, consideramos os vetores $\mathrm{N}=\{0,1,1,1,0\}$ e $\mathrm{C}=\{6,2,3,2,50\}$ e $\mathrm{w}=\{0.70,1.00,0.75,0.94,0.75\}$. Os atributos Resp.Time e Latency qualificam o recurso com valores baixos e Availability, Throughput e Reability qualificam com valores altos. Todos os atributos são normalizados com valor máximo definido em "C", o resultado é apresentado na Tabela 6.

A Tabela 7 apresenta os valores dos atributos após dividir o valor normalizado da Tabela 6 pelo maior valor normalizado de cada coluna. Após é somado todos os valores de atributos em cada linha. A seguir o recurso recebe um valor que vai de 0 a 100 . Será dado o valor 100 para o melhor recurso do dataset. A classificação de 1 a 4 é atribuída através da regra ilustrada na Figura 3. 
Tabela 5. Exemplo do dataset QWS

\begin{tabular}{lllll}
\hline Resp.Time & Availability & Throughput & Reliability & Latency \\
\hline 302.75 & 89 & 7.1 & 73 & 187.75 \\
482 & 85 & 16 & 73 & 1 \\
3321.4 & 89 & 1.4 & 73 & 2.6 \\
126.17 & 98 & 12 & 67 & 22.77 \\
107 & 87 & 1.9 & 73 & 58.33 \\
107.57 & 80 & 1.7 & 67 & 18.21 \\
255 & 98 & 1.3 & 67 & 40.8 \\
136.71 & 76 & 2.8 & 60 & 11.57 \\
102.62 & 91 & 15.3 & 67 & 0.93 \\
200 & 40 & 13.5 & 67 & 41.66 \\
\hline
\end{tabular}

Tabela 6. Atrib. Normalizados

Tabela 7. Resultado da Classificação

\begin{tabular}{|c|c|c|c|c|c|c|c|c|c|c|c|}
\hline & \multirow{2}{*}{\multicolumn{7}{|c|}{ MCDA }} \\
\hline RT & AV & TH & RE & $\mathbf{L A}$ & & & TH & $\mathbf{R E}$ & LA & MCDA & Classif \\
\hline 1.70 & 1.07 & 0.97 & 1.06 & 0.21 & 0.24 & 0.91 & 0.33 & 0.94 & 0.00 & 61.18 & 2 \\
\hline 1.07 & 1.02 & 2.19 & 1.06 & 38.56 & 0.15 & 0.87 & 0.75 & 0.94 & 0.70 & 85.99 & 1 \\
\hline 0.15 & 1.07 & 0.19 & 1.06 & 14.83 & 0.02 & 0.91 & 0.07 & 0.94 & 0.27 & 55.67 & 3 \\
\hline 4.07 & 1.18 & 1.64 & 0.98 & 1.69 & 0.57 & 1.00 & 0.56 & 0.86 & 0.03 & 76.42 & 1 \\
\hline 4.80 & 1.04 & 0.26 & 1.06 & 0.66 & 0.67 & 0.89 & 0.09 & 0.94 & 0.01 & 65.68 & 2 \\
\hline 4.78 & 0.96 & 0.23 & 0.98 & 2.12 & 0.67 & 0.82 & 0.08 & 0.86 & 0.04 & 62.27 & 2 \\
\hline 2.02 & 1.18 & 0.18 & 0.98 & 0.95 & 0.28 & 1.00 & 0.06 & 0.86 & 0.02 & 56.14 & 3 \\
\hline 3.76 & 0.91 & 0.38 & 0.87 & 3.33 & 0.53 & 0.78 & 0.13 & 0.77 & 0.06 & 57.22 & 3 \\
\hline 5.01 & 1.09 & 2.10 & 0.98 & 41.46 & 0.70 & 0.93 & 0.72 & 0.86 & 0.75 & 100 & 1 \\
\hline 2.57 & 0.48 & 1.85 & 0.98 & 0.93 & 0.36 & 0.41 & 0.63 & 0.86 & 0.02 & 57.59 & 3 \\
\hline
\end{tabular}

O algoritmo MCDA apresentado nesta seção foi utilizado para classificar o dataset contendo o repositório inicial de 100, 200 ou 300 recursos. Após 1000 recursos foram classificados e agrupados de diferentes formas para avaliar o comportamento do algoritmo de aprendizagem de máquina.

\subsection{Explorando aprendizagem de máquina na pré-classificação de recursos}

Esta seção apresenta o resultado de 4 avaliações realizadas na pré-classificação de recursos através do algoritmo de aprendizagem de máquina LMT. Foi escolhido o algoritmo LMT por ter obtido a melhor acurácia dentre os algoritmos de árvore de decisão analisados [Dilli et al. 2017]. Em cada avaliação foram classificados 1000 recursos com valores de 1 a 4. Os recursos foram agrupados em lotes de 50, 100, 200 e 500 recursos.

Todos os recursos foram classificados através do algoritmo MCDA e reclassificados de 1 a 4 . Essa reclassificação foi usada para treinar o algoritmo de aprendizado de máquina.

Em cada avaliação, o grupo de recursos classificados através do algoritmo MCDA é retirado do conjunto de dados de treinamento e usado no conjunto de dados de teste do algoritmo de aprendizado de máquina.

As avaliações foram realizadas com os seguintes repositórios iniciais para trei- 


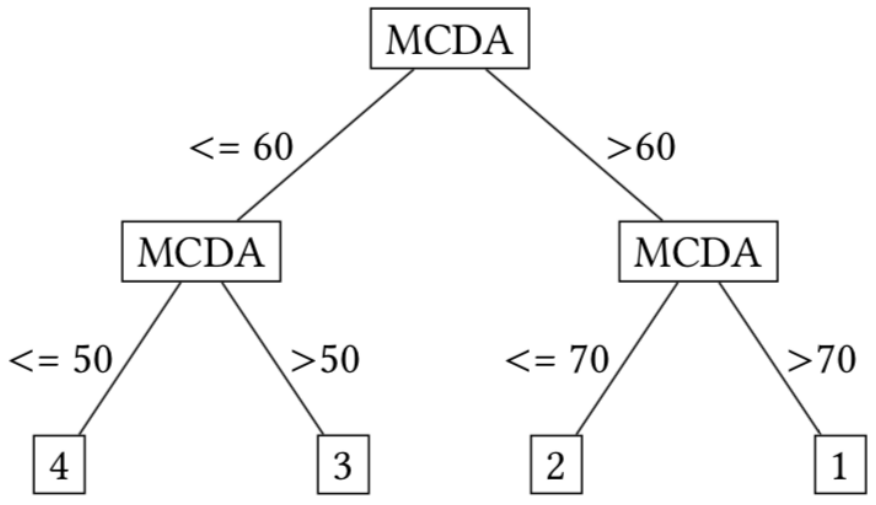

Figura 3. Reclassificação do algoritmo MCDA

namento do algoritmo LMT: (a) - repositório inicial com 100 recursos; (b) - repositório inicial com 200 recursos e (c) - repositório inicial com 300 recursos.

A Tabela 8 apresenta as classificações incorretas e o percentual de acurácia de cada uma das 4 avaliações:

Tabela 8. Avaliação da Acurácia

\begin{tabular}{lllllllllllll}
\hline Qty & E1a & E1b & E1c & E2a & E2b & E2c & E3a & E3b & E3c & E4a & E4b & E4c \\
\hline 50 & 14 & 7 & 8 & & & & & & & & & \\
100 & 13 & 8 & 10 & 26 & 21 & 21 & & & & & & \\
150 & 8 & 12 & 8 & & & & & & & & & \\
200 & 11 & 10 & 11 & 20 & 24 & 16 & 41 & 45 & 42 & & & \\
250 & 11 & 10 & 11 & & & & & & & & & \\
300 & 14 & 10 & 11 & 22 & 17 & 18 & & & & & & \\
350 & 7 & 6 & 10 & & & & & & & & & \\
400 & 11 & 8 & 10 & 13 & 14 & 15 & 35 & 34 & 28 & & & \\
450 & 5 & 6 & 6 & & & & & & & & & \\
500 & 6 & 6 & 5 & 14 & 12 & 13 & & & & 109 & 106 & 108 \\
550 & 7 & 6 & 14 & & & & & & & & & \\
600 & 12 & 14 & 8 & 16 & 14 & 27 & 63 & 61 & 77 & & & \\
650 & 20 & 21 & 6 & & & & & & & & & \\
700 & 17 & 19 & 9 & 35 & 38 & 16 & & & & & & \\
750 & 17 & 16 & 5 & & & & & & & & & \\
800 & 16 & 17 & 6 & 34 & 31 & 12 & 73 & 76 & 24 & & & \\
850 & 11 & 14 & 11 & & & & & & & & & \\
900 & 11 & 9 & 19 & 22 & 23 & 45 & & & & & & \\
950 & 14 & 10 & 7 & & & & & & & & & \\
1000 & 7 & 11 & 5 & 26 & 22 & 12 & 46 & 50 & 87 & 213 & 193 & 356 \\
\hline TOTAL & 232 & 223 & 180 & 228 & 216 & 195 & 258 & 266 & 258 & 322 & 299 & 464 \\
\hline Accuracy \% & 76.8 & 77.7 & 82 & 77.2 & 78.4 & 80.5 & 74.2 & 73.4 & 74.2 & 67.8 & 70.1 & 53.6 \\
\hline & & & & & & & & & & & & \\
\hline
\end{tabular}


A primeira avaliação (E1) considerou grupos de 50 recursos, totalizando 20 grupos. A segunda avaliação (E2) considerou grupos de 100 recursos, totalizando 10 grupos. A terceira avaliação (E3) considerou grupos de 200 recursos, totalizando 5 grupos. A quarta avaliação (E4) considerou grupos de 500 recursos, totalizando 2 grupos. Para cada grupo avaliado, o algoritmo MCDA reclassifica recursos e treina o algoritmo de aprendizado de máquina.

Ao analisar a acurácia obtida pelo pré-ranqueamento do algoritmo de aprendizado de máquina LMT, podemos observar que a avaliação E1c obteve a acurácia de $82 \%$. O repositório inicial foi definido com 300 recursos que foram usados para treinamento e lotes de 50 recursos para novos treinamentos.

As avaliações E2b e E2c também se mostram satisfatórias, com acurácia de 78.4\% e $80.5 \%$. Estas avaliações foram realizadas a cada 100 recursos, portanto os processos de normalização de dados e cálculos do algoritmo MCDA só é realizado a cada 100 recursos.

A avaliação 4 mostrou-se imprópria para a pré-classificação de recursos, com acurácia abaixo de 54\% (E4c). O intervalo de 500 recursos para recálculo do algoritmo MCDA e treinamento da aprendizagem de máquina mostrou-se muito grande, acarretando a perda de acurácia.

\section{Trabalhos Relacionados}

A Tabela 9 apresenta uma comparação dos principais Trabalhos Relacionados ao EXEHDA-RR, empregando os seguintes critérios: (i) expressividade na representação de recursos; (ii) utilização de algoritmos MCDA; (iii) aplicação das Preferências do Cliente; (iv) emprego de QoS; e (v) uso de lógica fuzzy e (vi) emprego de Aprendizagem de Máquina (AM). A presença do critério está representada pelo caracter "+" e a ausência por “_”.

Tabela 9. Comparação dos Trabalhos Relacionados

\begin{tabular}{lllllll}
\hline Trabalhos & Expr. & MCDA & Pref. & QoS & Fuzzy & AM \\
\hline Maheswari & + & + & + & + & + & - \\
Salah & - & + & + & + & + & - \\
Perera & + & + & + & - & - & - \\
Gomes & + & - & - & - & - & - \\
Almulla & - & + & + & + & + & - \\
Nunes & - & + & + & - & - & - \\
Suchithra & - & + & + & + & - & - \\
Vaadaala & - & + & + & + & - & + \\
EXEHDA-RR & + & + & + & + & + & + \\
\hline
\end{tabular}

A especificação de recursos através de linguagens de alta expressividade é realizada nos trabalhos de [Maheswari and Karpagam 2015], [Perera et al. 2013] e [Gomes et al. 2016].

Somente o trabalho de [Gomes et al. 2016] não considera as preferências do cliente, pois trabalha com dados de contexto.

Critérios de Qualidade de Serviço (QoS) são avaliados nos trabalhos 
de [Maheswari and Karpagam 2015], [Salah and Saadi 2016], [Almulla et al. 2015], [Suchithra and Ramakrishnan 2015] e [Vaadaala 2013].

A lógica fuzzy é discutida nos trabalhos [Maheswari and Karpagam 2015], [Salah and Saadi 2016] e [Almulla et al. 2015]. Nenhum deles aplica a lógica fuzzy para resolver a incerteza entre os especialistas.

Pode-se observar que apenas o trabalho de [Vaadaala 2013] utiliza aprendizagem de máquina. Mas com o objetivo de mensurar a acurácia de um único atributo de qualidade com o algoritmo J48. Os autores consideraram os resultados satisfatórios e obtiveram uma acurácia de $63 \%$.

\section{Considerações Finais}

Este artigo apresenta um modelo para classificação de recursos na IoT, denominado EXEHDA-RR. Como principal contribuição do trabalho desenvolvido até o momento, podemos destacar: (i) o uso da lógica fuzzy na definição dos pesos dos atributos e seu uso no algoritmo MCDA para classificar os recursos adequados à solicitação do cliente; e (ii) o uso do algoritmo de árvore de decisão na pré-classificação de recursos, considerando diferentes intervalos de treinamento.

Ao aplicar a lógica fuzzy na resolução de conflitos entre os especialistas na definição de graus de importância para cada atributo de QoS, foi possível promover a adequação dos pesos utilizados no algoritmo MCDA, considerando assim os recursos de melhor qualidade como resultado dos pedidos dos clientes.

Além disso, usando o aprendizado de máquina supervisionado, a partir de um conjunto de dados, classificado pelo algoritmo MCDA, foi possível classificar recursos com considerável acurácia. O algoritmo LMT foi adequado, com até 82\% de acurácia na pré-classificação.

O modelo de classificação de recursos proposto considera a dinamicidade da infraestrutura computacional fornecida pela IoT, com um grande número de consumidores e provedores de recursos.

O uso de algoritmos de aprendizado de máquina na pré-classificação de recursos promove uma redução relevante do esforço computacional dos algoritmos MCDA na classificação de recursos para cada solicitação dos clientes. Esta técnica foi modelada empregando tecnologias web semânticas para a especificação dos recursos e seus atributos de qualidade.

A perspectiva com a proposição do EXEHDA-RR é potencializar o processo de classificação de recursos do middleware EXEHDA, capacitando o mesmo para um cenário de elevada escalabilidade e dinamicidade quando da composição do ambiente computacional, situação esta típica da infraestrutura provida pela IoT.

Destacamos como trabalhos futuros a participação do usuário na definição dos pesos de importância dos atributos de QoS e a avaliação da acurácia na pré-classificação com datasets contendo um número maior de recursos. 


\section{Referências}

Al-Masri, E. and Mahmoud, Q. H. (2007). QoS-based discovery and ranking of Web services. In Proceedings - International Conference on Computer Communications and Networks, ICCCN, pages 529-534.

Almulla, M., Yahyaoui, H., and Al-Matori, K. (2015). A new fuzzy hybrid technique for ranking real world Web services. Knowledge-Based Systems, 77:1-15.

Argou, A., Dilli, R., Reiser, R., and Yamin, A. (2017). Lógica Fuzzy na Definição da Imortância dos Atributos de QoS na Seleção de Recursos da IoT. In IV WorkshopEscola de Informática Teórica, pages 173-180.

Davet, P. T. (2015). Uma Contribuicao à Inclusão Digital dos Métodos de Pesquisa Agropecuário no Cenário da IoT.

Dilli, R., Filho, H. K., Pernas, A. M., and Yamin, A. (2017). EXEHDA-RR: Machine Learning and MCDA with Semantic Web in IoT Resources Classification. WebMedia '17, October 17-20, 2017, Gramado, Brazil.

García, J. M. (2012). Improving Semantic Web Services Discovery and Ranking. PhD thesis, University of Seville.

Gomes, P., Cavalcante, E., Batista, T., Taconet, C., Chabridon, S., Conan, D., Delicato, F., and Pires, P. (2016). A QoC-Aware Discovery Service for the Internet of Things. Ubiquitous Computing and Ambient Intelligence, 7656:344-355.

IHS Markit (2017). The Internet of Things : a movement, not a market. Technical report.

Maheswari, S. and Karpagam, G. R. (2015). Comparative analysis of semantic web service selection methods.

Perera, C., Zaslavsky, A., Christen, P., Compton, M., and Georgakopoulos, D. (2013). Context-aware sensor search, selection and ranking model for internet of things middleware. In Proceedings - IEEE International Conference on Mobile Data Management, volume 1, pages 314-322.

Salah, N. B. and Saadi, I. B. (2016). Fuzzy AHP for Learning Service Selection in Context-Aware Ubiquitous Learning Systems. 2016 Intl IEEE Conferences on Ubiquitous Intelligence \& Comp., Adv. and Trusted Comp., Scalable Comp. and Communications, Cloud and Big Data Comp., Internet of People, and Smart World Congress, pages 171-179.

Schröpfer, C., Schönherr, M., Offermann, P., and Ahrens, M. (2006). A Flexible Approach to Service Management-Related Service Description in SOAs. In CEUR Workshop Proceedings, volume 234.

Suchithra, M. and Ramakrishnan, M. (2015). A survey on different web service discovery techniques.

Vaadaala, V. (2013). Classification of Web Services Using JForty Eight. A National Conference on Recent Trends in Computer Science and Technology International Journal of Electronics Communication and Computer Engineering, 4(6):181-184. 